\title{
An Investigation Into the Mechanism of Li Chong Pill for Treating Endometriosis Based on Network Pharmacology and Molecular Docking Verification
}

\section{Shao-Xuan Liu}

Heilongjiang University of Chinese Medicine https://orcid.org/0000-0002-4859-0121

Feng-Juan Han ( $\square$ hanfengjuan2004@163.com )

https://orcid.org/0000-0002-5953-3608

\section{Chun-Lan Zhang}

First Affiliated Hospital of Heilongjiang University of Chinese Medicine

\section{Ying Shen}

Heilongjiang University of Chinese Medicine

Jia Li

Heilongjiang University of Chinese Medicine

\section{Yang Fu}

First Affiliated Hospital of Heilongjiang University of Chinese Medicine

\section{Ying Guo}

First Affiliated Hospital of Heilongjiang University of Chinese Medicine

\section{Research}

Keywords: Endometriosis, Network pharmacology, molecular docking, Li Chong Pill

Posted Date: March 8th, 2021

DOI: https://doi.org/10.21203/rs.3.rs-281498/v1

License: (c) (7) This work is licensed under a Creative Commons Attribution 4.0 International License. Read Full License 


\section{Abstract}

Background and objective: Li Chong Wan (Li Chong pill, LCP) origin from Yi Xue Zhong Zhong Can Xi Lu, (Records of Chinese Medicine with Reference to Western Medicine), widely used in the treatment of endometriosis (EM) in China. The purpose of this study is to investigate the intrinsic mechanisms of LCP against EM and to provide new evidence for its clinical application.

Methods: Chemical compounds of LCP were screened and evaluated via retrieving public databases and literature. We also acquired their putative targets and obtained EM-related targets. The above-mentioned data were visualized as a component-target network. In addition, we use Cytoscape3.8.0 to build a protein-protein interaction network and identified hub genes and key active ingredients. Furthermore, through GO and KEGG pathway analyses, which were actualized by R3.6.1 (based on clusterProfiler, org.Hs.eg.Db, and pathview package), we obtained effective signaling pathways and biological functions. Molecular docking was used to verify binding activity between compounds and the key targets at last.

Results: Finally, a total of 122 possible active targets and 47 components were screened. Identify the core network and screen out 10 main targets; GO and KEGG enrichment analysis revealed that LCP may have functions of antiinflammatory, anti-angiogenesis, inhibition of cell proliferation, regulation of hormone secretion, etc. The effect of LCP on EM might be achieved by PI3K/Akt signaling pathway, HIF-1 signaling pathway, estrogen signaling pathway, and VEGF signaling pathway, etc. Finally, molecular docking results demonstrated that 14 components were exhibited good binding property to the key targets of EM.

Conclusion: This research ocularly demonstrated the multi-component, multi-target, and multi-channel pharmacological effects for LCP in the treatments of EM and provides evidence for further clinical research and verification of the mechanism.

\section{Introduction}

Endometriosis is estimated to affect $10 \%-15 \%$ of women at childbearing age. ${ }^{1}$ The endometriotic pathological processes involve repeated tissue injury and repair, local inflammation, angiogenesis, and neurogenesis $\varangle$ which result in progressive smooth muscle metaplasia and uterine fibrogenesis. ${ }^{2}$ The preferred treatment is surgery. However, surgery carries a significant recurrence rate and cost burden. There is a critical need for safe, effective medical therapies for endometriosis patients at present, either in conjunction with or independent of surgery. ${ }^{3}$ And an important fact is that the increased risk of deeply infiltrating endometriosis in patients is associated with surgical history. ${ }^{4}$ It might be related to activation of the adrenergic pathway, chronic stress, and increased angiogenesis. ${ }^{5}$ Traditional Chinese medicine has a unique view on the treatment of endometriosis. It is believed that the basic etiology of this disease is "accumulated static blood in the uterus", and blood stasis is the central link running through EM and the most basic pathological basis of the disease. ${ }^{6}$

Li Chong Wan (Li Chong pill, LCP) origin from Yi Xue Zhong Zhong Can XiLu, (Records of Chinese Medicine with Reference to Western Medicine), which is composed of Shuizhi (Leech), Huangqi (Astragalus), Sanleng (Sparganii Rhizoma), Ezhu (Curcumae Rhizoma), Danggui (Chinese Angelica), Zhimu (Anemarrhena) and Taoren (Peach kernel). It is found that these drugs have the effect of anti-inflammatory, analgesic, hemodynamic improvement, and antioxidative stress. ${ }^{7-12}$ In addition to the widespread use of endometriosis, LCP is also used to treat all kinds of benign abdominal masses of women, such as ovarian cysts, old ectopic pregnancy and so on. ${ }^{13,14}$ The pathological mechanism of EM is complex and the symptoms are diverse, while the traditional Chinese medicine compound is 
made up of many natural herbs, which has the characteristics of multi-component, multi-target, multi-function, and multi-pathway. Network pharmacology is a part of bioinformatics, which uses drug research models by constructing drug-drug, drug-target networks to find interactions between bioactive compounds and targets and between various targets, ${ }^{15}$ and molecular docking is a method which can predict ligand-target interactions at a molecular level, ${ }^{16}$ widely used in drug discovery. Therefore, we apply this method to explore the relationship between LCP and EM. The relevant technology route is shown in Figure 1.

\section{Materials And Methods}

\section{Data preparation}

\section{Construction of LCP chemical ingredients database}

Except for leech, all chemical ingredients of the other six drugs in LCP were coming from TCMSP (Traditional Chinese Medicine Systems Pharmacology Database and Analysis Platform, https://tcmspw.com/tcmsp.php), a website that integrates computer systems and biological sciences, with the primary objective of helping understand how drugs work in specific pathways and different cell types. It contains 499 Chinese medicines registered in the Pharmacopoeia of the people's Republic of China, and including 29,384 chemical ingredients, 3311 targets, and 837 related diseases, it also provides ADME-related properties data to undertake the discovery and research of drugs. ${ }^{83}$ We used oral bioavailability (OB), drug-like properties (DL) in the TCMSP database as the index to select the target active components $(O B \geq 30 \%$ and $D L \geq 0.18 \%)$ referring to similar literature, ${ }^{84}$ finally obtained the active ingredients of Huangqi (Astragalus), Sanleng (Sparganii Rhizoma), Ezhu (Curcumae Rhizoma), Danggui (Chinese Angelica), Zhimu (Anemarrhena) and Taoren (Peach kernel). Moreover, the chemical constituents of leech were screened out from exploring literature in CNKI (https://www.cnki.net/) and PubMed (https://pubmed.ncbi.nlm.nih.gov/). Then we used the PubChem database (https://pubchem.ncbi.nlm.nih.gov/) to find canonical simplified molecular-input entry specification (SMILES) information of those constituents and used Chem Bio Draw Ultra software for drawing the 2D structures of the candidate compounds not found in PubChem. Then upload them to the Swiss ADME (http://www.swissadme.ch/), a website that can evaluate pharmacokinetics, drug-likeness, and medicinal chemistry friendliness of small molecules. An active compound can be screened out when the following conditions are met simultaneously, firstly, using the Pharmacokinetics Gatrointesinal absorption which for screening active compounds with good oral bioavailability shows "High" as a condition that drugs can be absorbed, secondly, there are 2 or more "Yes" in the 5 drug-like predictions (Lipinski, Ghose, Veber, Egan, Muegge) results.

\section{LCP-related Target prediction}

First, using TCMSP to predict protein targets of six drugs except for leeches, using UniProt KB

(http://www.uniprot.org) to extract the predicted targets, convert them into symbol names, the target information was set to homo sapiens and consolidate the downloaded data for further analyses. Secondly, the results of leech active components screened by Swiss ADME were uploaded to the Swiss Target Prediction (https://www.swisstargetprediction.ch/), setting probability $>0.1$ (Probability for the query molecule -assumed as bioactive to have this protein as the target.) was used to screen the target of the leech, so as to establish the drug target database of LCP.

\section{Endometriosis Related Genes Database Construction}


Obtained EM-related targets through five databases which were Gene Cards, OMIM, TTD, PharmGKB, and Drug Bank. Gene Cards integrated gene databases in a variety of research areas, including genomes, transcriptomics, proteomics, which is a one-step-shop for searchable human gene annotations. ${ }^{85}$ OMIM (Online Mendelian Inheritance in Man www. omim.org) provides concise textual information based on the peer-reviewed biomedical literature on over 15,500 genes, 26,200 allelic variants, and 7,800 genetic phenotypes. ${ }^{86}$ TTD (Therapeutic Target Database), which enables the search of almost 900 targets, 1800 biomarkers, and 6000 drugs related to 900 diseases conditions. ${ }^{87}$ Drug Bank is a fully curated drug and drug target database. ${ }^{88}$ And PharmGKB today is the preeminent worldwide resource for pharmacogenomic information. ${ }^{89}$ We searched the above-mentioned database using the keyword "endometriosis", the gene data obtained from the search were summarized and the duplicated targets were removed for subsequent analysis.

\section{Construction of an LCP-compound-target-EM Network}

We map the Venn between gene and drug targets via the "venn" R package disease. And the LCP-compound-targetEM network was constructed to clarify the relationship between active compounds in LCP and potential targets. This network was constructed and visualized using Cytoscape 3.8.0 software.

\section{Protein-protein Interaction Network Between Targets}

Since protein-protein interactions (PPI) are associated with the development of disease states, ${ }^{90}$ we introduce potential targets into the STRING database (https://string-db.org/) for PPI analysis. STRING is an online PPI analysis database that aims to collect, score and integrate all publicly available sources of PPI information, and construct a visualized interaction network of genome-wide datasets. ${ }^{91}$ The species was limited to "Homo sapiens", and the minimum required interaction score was set to be high confidence (0.900). We used the STRING database to explore the protein interaction relationship between LCP and endometriosis and map the protein interaction network.

\section{GO and KEGG enrichment analysis}

To explore the enrichment in gene function of interaction proteins obtained from the above analysis, we used R3.6.1 (based on "clusterProfiler"'"org.Hs.eg. db" and" pathview" package) to analyze the biological enrichment of GO and KEGG.

\section{Verification through Molecular Docking}

The Protein Data Bank (PDB), the single global repository of experimentally determined 3D structures of biological macromolecules and their complexes, was established in 1971, becoming the first open-access digital resource in the biological sciences. The PDB archive currently houses 130,000 entries (May 2017). ${ }^{92}$ Therefore, we searched the PDB database for the 3D structures of the significant potential targets of the protein-protein interaction network, apply PyMOL2.4.0 to modify the target protein, including removal of ligands and water. Select the active ingredients whose degree $>5$ in the LCP-compound-target-EM network, use the PubChem to download the 2D structure of those components, and use the ChemDraw to draw things that are not found. Apply the MM2 in the Chem3D to optimize the energy of molecular configuration. Finally, we used AutoDock Tools1.5.6 to optimize the structure of protein molecules and ligands. Upload the results to CB-Dock (http://cao.labshare.cn/cb-dock/), a website that automatically determines the active pocket and sets the active site size and center coordinates. AutoDock vina software is used to operate molecular docking, which can also calculate the best binding energy of the optimal docking mode. 


\section{Result}

\section{Active compounds of the LCP}

A total of 98 components were screened from LCP. Among the active ingredients screened from the TCMSP, we selected 68 ingredients according to OB and DL (see Additional file 1), including Huangqi (astragalus, HQ) 20 species, Taoren (Peach kernel TR) 23 species, Zhimu (anemarrhena, ZM)15 species, Ezhu (Curcumae Rhizoma, EZ) 3 species, Sanleng (Rhizoma sparganii, SL) 5 species, Danggui (Chinese angelica, DG) 2species. In addition, we upload 50 (see Additional file 2) compounds of leech obtained from document retrieval. ${ }^{17-21}$ According to the two conditions, we get 30 active ingredients of leech in the end (see Additional file 3 ).

\section{Putative targets of $L C P$}

According to the above active ingredients,881 potential targets were to predicted by using TCMSP and Swiss Target Prediction, the targets included 209 from HQ, 59 from TR, 85 from ZM, 9 from EZ, 54 from SL,29from DG, 436 from SZ (see Additional file 4), gathering them up and removed the duplicate targets, the final number was 380.

\section{EM related targets}

Endometriosis-related genes, obtained from five databases previously described, we get 90 genes from the Drug Bank, 1009 genes from the GeneCards, two genes from the OMIM, 86 genes from the PharmGKB, 15 genes from the TTD, a total of 1115 targets were obtained after sorting out the screening data (Figure 2 ).

\section{Construction of LCP-compound-target-EM Network}

From the Venn chart, we found there were 122 overlapping targets between drugs and disease potential targets (Figure 3). Use Cytoscape3.8.0 to map the LCP-compound-target-EM network (Figure 4), there are 168 nodes (Except overlapping targets, 45 nodes are drug constituents, including 12 from TR, 10 from SZ,7 from ZM, 11 from HQ, 1 from SL, and A1, B1, C1, D1, E1 means the ingredient comes from a variety of drugs, see Table 1) and edge 298 in the network》It can be seen that the effects of traditional Chinese medicines are widely distributed, each ingredient in LCP can take part in the treatment of diseases through a synergistic action.

\section{PPI Network Analysis and Hub Genes Investigation}

122 potential targets were uploaded to the STRING database. High confidence will improve the accuracy of the results so we set the minimum required interaction score to "highest confidence" $(0.900)$. There were 110 proteins in the network, which contained 371 protein interaction relationships (Figure 5), and then filtered with application CytoNCA ${ }^{22}$, a Cytoscape plugin for centrality analysis and evaluation of protein interaction networks (Figure 6a), based on the median values for betweenness, closeness, degree, eigenvector, LAC and network, which were39.31546869, 0.1244298725, 6, 0.0291560585, 2.5333333335, 3.4583333335, identified 28 highly connected node (Figure 6b), then performed second screening, which was 9.1005245735,0.586956522,9,0.1712328495, $4.211111111,5.154761905$. We gradually obtained the core network of hub genes, finally identified 10 highly connected nodes significant endometriosis-related targets (Figure 6c), which are MAPK1, FOS, JAK2, EGFR, RELA, SRC, MAPK8, PIK3CA, MAPK14, and ESR1. (Figure 6d)

\section{GO and KEGG enrichment analysis}


The Gene Ontology (GO) can describe how genes act in biological systems and produce a dynamic, controlled vocabulary that can be applied to all eukaryotes, ${ }^{23}$ which concludes three parts, biological progress (BP), cellular compound (CC), and molecular function (MF) to describe the biochemical activity of a gene product. Kyoto Encyclopedia of Genes and Genomes (KEGG) is a knowledge base for systematic analysis of gene functions in terms of the networks of genes and molecules, which can provide most of the known metabolic pathways and some of the known regulatory pathways. ${ }^{24}$ We used R packets to perform GO and KEGG enrichment analysis of 122 intersected genes, the detailed results are listed in additional files (see Additional file 5 and Additional file 6), the top 10 GO enrichment results of each project are shown in figure 7a/b. At the top of the BP group was "response to nutrient levels", "cellular response to chemical stress", "reproductive structure development", "reproductive system development", "response to oxygen levels" and "response to oxidative stress", etc. The MF group mainly included "steroid binding", "protein tyrosine kinase activity", "nuclear receptor activity" and "ligand-activated transcription factor activity", etc. And in the CC group, the GO terms were mainly involved "membrane raft", "membrane microdomain", "membrane region", "organelle outer membrane", "outer membrane mitochondrial outer membrane" and so on, which reflected the abnormality of multiple biological processes involved in endometriosis, and indicates that LCP may play a therapeutic role by improving the above biological pathways.

The KEGG pathway enrichment shows the top 30 contains, (Figure 8 a/b.) such as PI3K-Akt signaling pathway, EGFR tyrosine kinase inhibitor resistance, Th17 cell differentiation, relaxin signaling pathway, HIF-1 signaling pathway, Ovarian steroidogenesis, PD-L1 expression, and PD-1 checkpoint pathway in cancer and so on. The results of the first 30 of the GO and KEGG enrichment analysis were made into bubble charts and histograms, and produced a pathway map that the gene most enriched.

\section{Verification of Molecular Docking}

We screened 14 key components according to the LCP-compound-target-EM network(degree>5) as small-molecule drug ligands, which were docked with the 10 key targets identified in the previous PPI network (Table 2), then we obtained 140 sets of results(Table 3). The binding energy<0, indicating that the ligand molecules can bind spontaneously to the receptor proteins, however, there was no clear definition of the criteria for free energy screening after searching the literature $\bigotimes$ This paper take the binding energy $<-5.0 \mathrm{kal}$ ' mol-1 as a standard to judge the binding property is good, and the smaller the binding energy, the better running of docking. We found that the main way of molecules docking were hydrogen bonding and $\pi-\pi$ stacking, The minimum binding energy is -10.5 kal ${ }^{\circ}$ mol- 1 (Figure 9), and the binding energy $<-5.0 \mathrm{kal}{ }^{\circ}$ mol-1 accounts for $96.43 \%$ of the total, and the binding energy $>-5.0 \mathrm{kal}^{\circ} \mathrm{mol}^{-1} \mathrm{was}^{-}$ abandoned. It can fully explain that the pivotal components of LCP have strong binding force with their key targets.

Table 1 Description of nodes in the LCP-compound-target-EM Network (Figure 3) 


\begin{tabular}{|c|c|c|}
\hline $\begin{array}{l}\text { Node } \\
\text { Name }\end{array}$ & Source & Compound \\
\hline A1 & Zhimu,Danggui,Sanleng & Stigmasterol \\
\hline B1 & Taoren,Danggui,Sanleng & Beta-sitosterol \\
\hline C1 & Huangqi, Sanleng & Formononetin \\
\hline D1 & Huangqi, Zhimu & Kaempferol \\
\hline E1 & Huangqi,Taoren,Sanleng,Ezhu & Hederagenin \\
\hline HQ1 & Huangqi (astragalus,HQ) & Bifendate \\
\hline HQ2 & Huangqi (astragalus,HQ) & Jaranol \\
\hline HQ3 & Huangqi (astragalus,HQ) & Calycosin \\
\hline HQ4 & Huangqi (astragalus,HQ) & $\begin{array}{l}\text { (3S,8S,9S,10R,13R,14S,17R)-10,13-dimethyl-17-[ (2R,5S)-5-propan-2- } \\
\text { yloctan-2-yl]-2,3,4,7,8,9,11,12,14,15,16,17-dodecahydro-1H- } \\
\text { cyclopenta[a]phenanthren-3-ol }\end{array}$ \\
\hline HQ5 & Huangqi (astragalus,HQ) & FA \\
\hline HQ6 & Huangqi (astragalus,HQ) & Quercetin \\
\hline HQ7 & Huangqi (astragalus,HQ) & Isorhamnetin \\
\hline HQ8 & Huangqi (astragalus,HQ) & $\begin{array}{l}\text { (6aR,11aR)-9,10-dimethoxy-6a,11a-dihydro-6H-benzofurano[3,2- } \\
\text { c]chromen-3-ol }\end{array}$ \\
\hline HQ9 & Huangqi (astragalus,HQ) & Mairin \\
\hline HQ10 & Huangqi (astragalus, HQ) & 7-0-methylisomucronulatol \\
\hline HQ11 & Huangqi (astragalus,HQ) & 3,9-di-O-methylnissolin \\
\hline SL1 & Sanleng (leech, SZ) & trans-gondoic acid \\
\hline SZ1 & Sanleng (leech, SZ) & gamma-aminobutyric acid \\
\hline SZ2 & Sanleng (leech, SZ) & L-tryptophan \\
\hline SZ3 & Sanleng (leech, SZ) & phenylalanine \\
\hline SZ4 & Sanleng (leech, SZ) & hexadecyl ethers of glycerol \\
\hline SZ5 & Sanleng (leech, SZ) & methyl 14-methylpentadecanoate \\
\hline SZ6 & Sanleng (leech, SZ) & L-tyrosine \\
\hline SZ7 & Sanleng (leech, SZ) & methyl (Z)-11-hexadecenoate \\
\hline SZ8 & Sanleng (leech, SZ) & hirudinoidine a \\
\hline SZ9 & Sanleng (leech, SZ) & palmitic acid \\
\hline SZ10 & Sanleng (leech, SZ) & glycerol \\
\hline TR1 & Taoren (peachkernel, TR) & Gibberellin A44 \\
\hline TR2 & Taoren (peach kernel, TR) & 3-0-p-coumaroylquinic acid \\
\hline
\end{tabular}




\begin{tabular}{|c|c|c|}
\hline TR3 & Taoren (peach kernel, TR) & 2,3-didehydro GA70 \\
\hline TR4 & Taoren (peach kernel, TR) & 2,3-didehydro GA77 \\
\hline TR5 & Taoren (peach kernel, TR) & GA120 \\
\hline TR6 & Taoren (peach kernel, TR) & GA63 \\
\hline TR7 & Taoren (peach kernel, TR) & $\begin{array}{l}\text { 4a-formyl-7alpha-hydroxy-1-methyl-8-methylidene-4aalpha,4bbeta- } \\
\text { gibbane-1alpha,10beta-dicarboxylic acid }\end{array}$ \\
\hline TR8 & Taoren (peach kernel, TR) & Sitosterol alpha1 \\
\hline TR9 & Taoren (peach kernel, TR) & GA60 \\
\hline TR10 & Taoren (peach kernel, TR) & GA121-isolactone \\
\hline TR11 & Taoren (peach kernel, TR) & GA77 \\
\hline TR12 & Taoren (peach kernel, TR) & campesterol \\
\hline ZM1 & Zhimu (anemarrhena, ZM) & Timosaponin B III_qt \\
\hline ZM2 & Zhimu (anemarrhena, ZM) & Anemarsaponin F_qt \\
\hline ZM3 & Zhimu (anemarrhena, ZM) & Hippeastrine \\
\hline ZM4 & Zhimu (anemarrhena, ZM) & Anemarsaponin C_qt \\
\hline ZM5 & Zhimu (anemarrhena, ZM) & coumaroyltyramine \\
\hline ZM6 & Zhimu (anemarrhena, ZM) & Anhydroicaritin \\
\hline ZM7 & Zhimu (anemarrhena, ZM) & diosgenin \\
\hline
\end{tabular}

Table 2 The PDB ID corresponding to each core target in the PPI network

\begin{tabular}{|ll|}
\hline Target & PDB ID \\
\hline JAK2 & $3 U G C$ \\
\hline MAPK1 & $6 S L G$ \\
\hline FOS & $1 \mathrm{AO} 2$ \\
\hline EGFR & $2 \mathrm{MOB}$ \\
\hline RELA & $3 \mathrm{QXY}$ \\
\hline SRC & $2 \mathrm{H} 8 \mathrm{H}$ \\
\hline MAPK8 & $2 \mathrm{NO} 3$ \\
\hline PIK3CA & $6 \mathrm{PYS}$ \\
\hline MAPK14 & $2 \mathrm{LGC}$ \\
\hline ESR1 & $2 \mathrm{BJ} 4$ \\
\hline
\end{tabular}

Table 3 Docking scores of the active ingredients of LCP with their potential targets 


\begin{tabular}{|c|c|c|c|c|c|c|c|c|c|c|}
\hline Compound & $3 U G C$ & $6 S L G$ & $1 \mathrm{AO} 2$ & $2 \mathrm{MOB}$ & $3 Q X Y$ & $2 \mathrm{H} 8 \mathrm{H}$ & $2 \mathrm{NO} 3$ & 6PYS & 2LGC & $2 \mathrm{BJ} 4$ \\
\hline Quercetin & -7.8 & -7.9 & -8.4 & -6.5 & -9.2 & -9.2 & -9 & -8.6 & -8.6 & -8.1 \\
\hline Hirudinoidine a & -7.2 & -7 & -7.6 & -6 & -7.4 & -7.3 & -7.6 & -7.5 & -7.5 & -6.2 \\
\hline $\begin{array}{l}\text { Methyl (Z)-11- } \\
\text { hexadecenoate }\end{array}$ & -6.4 & - & -5.5 & - & -6.5 & -6.1 & -5.6 & -5.9 & -7.1 & -6.2 \\
\hline $\begin{array}{l}\text { Methyl } 14- \\
\text { methylpentadecanoate }\end{array}$ & -6.3 & - & -5.9 & - & -6.5 & -5.6 & -5.5 & -6.1 & -7.1 & -5.9 \\
\hline Kaempferol & -9 & -7.9 & -8.1 & -6.3 & -9.4 & -9.1 & -8.9 & -8.3 & -8.5 & -8.3 \\
\hline Isorhamnetin & -8.8 & -7.9 & -9.8 & -6.3 & -9.2 & -9.4 & -8.7 & -8.5 & -8.4 & -8.2 \\
\hline Beta-sitosterol & -8.9 & -9.2 & -8.1 & -7 & -8.2 & -9.5 & -9 & -8.4 & -9 & -7.8 \\
\hline Stigmasterol & -9.5 & -9.8 & -8.2 & -6.8 & -9.1 & -9.1 & -9.3 & -9 & -8.3 & -7.2 \\
\hline $\begin{array}{l}\text { 7-0- } \\
\text { methylisomucronulatol }\end{array}$ & -7.5 & -7 & -7.4 & -6.6 & -9 & -8.4 & -7.5 & -7.1 & -8.2 & -7 \\
\hline Anhydroicaritin & -8.4 & -8.2 & -8.7 & -6.3 & -9.3 & -9.4 & -8.8 & -9.5 & -7.8 & -8.3 \\
\hline Formononetin & -8.8 & -7.8 & -7.9 & -6.5 & -9.2 & -9.2 & -8.7 & -9 & -9 & -8.6 \\
\hline Calycosin & -8.3 & -7.9 & -7.7 & -6.4 & -9.3 & -9.1 & -8.7 & -9.3 & -8.9 & -8.2 \\
\hline Diosgenin & -9.6 & -9.4 & -9.2 & -7.2 & -9.1 & -10.4 & -9.2 & -9.8 & -10.5 & -8.1 \\
\hline phenylalanine & -6.4 & -5.5 & -6.2 & - & -6.3 & -6.4 & -5.9 & -6.6 & -6.5 & -6.2 \\
\hline
\end{tabular}

\section{Discussion}

Abnormally located endometrium can grow and shed during the menstrual cycle, causing painful menstrual cramps or heavy menstrual periods. The development of inflammation, cysts, scar tissue, and adhesions leading to intestinal problems, chronic pelvic inflammatory disease, and infertility. ${ }^{25}$ The problems caused by EM have brought great pressure to women's psychology and life, research on the disease has become a hot area in gynecology. Modern medicine suggests that the occurrence of EM possibly related to1) Retrograde menstruation 2) Coelomic metaplasia and 3) Vascular and lymphatic metastatic spread. ${ }^{26}$ Besides, studies have shown that the growth of ectopic endometrial tissue leads to the recruitment of a large number of immune cells and the abnormal function of almost all immune cells, which increase pro-inflammatory cytokines, growth factors, and acceleration of angiogenesis. These mechanisms aggravate the inflammatory response of local tissues. ${ }^{27}$ In a review in 2019 that proposed a concept named "endometriosis life", ${ }^{2}$ the literature suggests that doctors should first consider addressing pelvic pain or providing assisted reproduction technologies rather than surgery immediately in cases of EM. Traditional Chinese medicine also has made a lot of research and clinical observation in the treatment of it, based on the complex mechanism of EM, it can provide multi-target treatment and new ideas for clinical treatment of this disease.

LCP is one of the classical prescriptions of traditional Chinese medicine for EM, modern medicine made a more careful study of drugs it contains. In this research, diosgenin, kaempferol, quercetin, stigmasterol, isorhamnetin, betasitosterol, formononetin, anhydroicaritin, calycosin, hirudinoidine a, and 7-0-methylisomucronulatol were the compounds that degree $>5$ of the LCP-compound-target-EM network and performed well in subsequent molecular docking. As we can see from the molecular docking results, the best performing component is diosgenin, which from 
the Zhimu, it's a steroid sapogenin found in several plants which possesses a wide range of biological activities. ${ }^{28}$ First of all, it has good anti-inflammatory activity, ${ }^{29}$ there were some researches demonstrated that diosgenin significantly inhibited leukocyte migration and adhesion which was partly linked with the downregulation of TNF-ainduced expression of ICAM-1 via inhibiting NF-KB p65 activation. It also can inhibit the up-regulation of adhesion molecules induced by TNF-a, through the inhibition of MAPK/Akt and NF-KB signaling pathways and ROS production. ${ }^{30,31}$ An experiment indicated that diosgenin may be an effective NADPH oxidase inhibitor that can restrain superoxide anion production to play an anti-inflammatory role which is associated with inhibition of the cPLA2, PAK1/2, Akt, p38MAPK, ERK1/2, and JNK signaling pathway. ${ }^{28}$ Secondly, ectopic endometrial lesions not only induce a local inflammatory response but also accompanied by abnormal coagulation function, ${ }^{32}$ meanwhile, oxidative stress is involved in the pathogenesis of EM through various mechanisms, which is one of the probable mechanisms leading to carcinogenesis and infertility of patients. ${ }^{33}$ Experiments prove diosgenin has anti-thrombosis properties, ${ }^{34}$ the cytoprotective effect on vascular endothelial cells. ${ }^{30}$ It also can modulate antioxidant defense and prevent endothelial apoptosis under oxidative stress, ${ }^{35}$ At last, an experimental result showed that diosgenin can increase mechanical and thermal nociceptive thresholds and lowered pain scores in diabetic rats through lowering oxidative stress and inflammation and improving antioxidant defense system. ${ }^{36}$ We can speculate that diosgenin can alleviate the pain caused by EM, in the same way, which needs deep and extensive clinical trials to prove. Of all the docking results, the binding energies of diosgenin act on MAPK14, SRC, and PIK3CA were - 10.5 kal ' mol-1, $-10.4 \mathrm{kal}$ ' mol-1, and $-9.8 \mathrm{kal}{ }^{\prime} \mathrm{mol}-1$ respectively. The first two have been shown to be associated with inflammatory disease and angiogenesis. ${ }^{37,38}$ PIK3CA is one of the most common mutation genes found in tumor types in recent years. ${ }^{39}$ It is reported that mutations in this gene involve the occurrence of ovarian cancer in EM. ${ }^{40}$ Hence, in addition to the above analysis, diosgenin may play a therapeutic role mainly through these three targets. In summary, we can see that diosgenin can affect EM in various aspects. Besides, quercetin is one of the important ingredients too, it has anti-proliferative and anti-inflammatory effects on endometriosis auto-implanted mouse models, this ingredient not only can induce ectopic endometrial atrophy ${ }^{41}$ but also inhibited the proliferation and induced the cell cycle arrest in VK2/E6E7 and End1/E6E7 cells. It also can induce cell apoptosis by DNA fragmentation, reactive oxygen species production, and loss of mitochondrial membrane potential. ${ }^{42}$ The main components selected in LCP also include kaempferol, which can decrease cell trauma rely on reducing the inflammatory mechanism caused by oxidative stress, and could have anti-inflammatory effects with inhibiting the expression of MAPK signaling pathway and inhibited some inflammatory mediators. ${ }^{43}$ Isorhamnetin, too, has distinct anti-inflammatory properties, ${ }^{44}$ it can inhibit the inflammation, proliferation, and migration of BEAS-2B cells by regulating the MAPK and NF-KB signaling pathways and is a drug candidate for asthma. ${ }^{45}$ According to the above analysis. Active compounds of LCP may play a major role in the treatment of EM with the function of anti-inflammatory, anti-oxidative stress, antiangiogenesis, smoothing of pain, and promoting ectopic tissue atrophy.

We screened out 10 major potential targets after Cytoscape3.8.0 analysis to unraveling the complex molecular relationships, emphasizing the regulatory role of LCP in endometriosis through this part of the target. RELA is one of the core targets selected by the PPI. Although there are many dimer forms of NF-kB, the main form is still p50and p65/RELA subunits, encoded by the NFKB1 and RELA genes. ${ }^{46}$ NF-KB is a major transcription factor in apoptosis and cell growth processes that regulates the expression of genes and molecules, a study showed that inhibition of NF$\mathrm{KB} /$ relA activity in ovarian cancer cells can suppress angiogenesis and progressive growth. ${ }^{47}$ In endometriosis, stimulation of pro-inflammatory cytokines such as IL-6, Tumor necrosis factor a (TNFa), and IL-1 $\beta$ induce nuclear factor-KB (NF-KB) activation, which in turn, stimulates the production of a wide spectrum of pro-inflammatory cytokines, resulting in constant NF-KB activation and a cascade of downstream changes in the cells. ${ }^{46}$ Furthermore,

Page $10 / 28$ 
the results of molecular docking showed that RELA had good binding activities to each active ingredient, it is not difficult to conjecture that LCP may achieve the purpose of anti-inflammatory by inhibiting the NF-KB signaling pathway. Epidermal growth factor (EGF) is immunolocalized in both glandular epithelia and stroma of endometriotic tissue. Compared to women without this disease, a marked increase in EGF is detected in women with endometriosis. ${ }^{48,49}$ EGF increased the expression of endometriosis-associated hyaluronan and its synthase HAS2, both of which mediated EGF-induced stromal cell migration and invasion in women with EM. ${ }^{50}$ Dysregulation of Matrix metalloproteinases (MMPs) is related to endometriosis risk, ${ }^{51}$ EGF receptor (EGFR) inhibitor treatments regressed endometriotic lesions, and decreased MMP-7 activities in a mouse model of endometriosis. ${ }^{52}$ And the good molecular docking results suggested that the active components of LCP can inhibit the proliferation of ectopic tissue by depressing the activity of EGFR, to achieve the therapeutic effect. Another core target we have identified is FOS, FOS is a nuclear phosphoprotein, encoded by mature m RNA from gene transcription of c-fos. ${ }^{53}$ EM is an estrogendependent gynecological disease, ${ }^{54} \mathrm{C}$-fos gene expression presented a significant correlation with the circulating estradiol concentrations, ${ }^{55}$ and there was an experiment further demonstrated that $17 \beta$-E2-induced human endometrial stromal cell (HESC) invasion is dependent on c-fos mediated MMP-9 expression. ${ }^{56}$ Therefore, it is speculated that LCP can achieve the purpose of treatment by inhibiting c-FOS expression. Moreover, the ratio of ESR1 to ESR2 of ectopic lesions in patients with EM was lower than eutopic endometrium, excessive levels of ESR2 mediates an estrogen-driven inflammatory process and prostaglandin formation. ${ }^{57}$ Our molecular docking results showed that each active ingredient had a degree of regulatory effect on the estrogen receptor 1 (ERS1) gene.

By exploring the results of KEGG enrichment, we conclude that the signaling pathway related to endometriosis involves cell proliferation and apoptosis, immune response, sex hormone regulation, angiogenesis and pain regulation, etc. A large number of genes were associated with $\mathrm{PI} 3 \mathrm{~K} / \mathrm{Akt}$ signaling pathways (Figure 10). Research shows that this pathway is significantly expressed in endometriosis. ${ }^{58} \mathrm{PI} 3 \mathrm{~K}$ s is involved in regulating different cellular functions including inflammation, auto-immune disorders, cell apoptosis, and cancer progression, 59-63 phosphoinositol-dependent protein kinase 1 (PDK1) can be activated by the phosphoinositide-3,4,5- triphosphate (PIP3) which generated from PI3K囚those changes can activate the serine/threonine kinase Akt by phosphorylating its kinase domain, ${ }^{64}$ a study shows that inhibition of the PI3K/Akt/mTOR signaling pathway attenuates the inflammatory response in rats with osteoarthritis. ${ }^{65}$ Active Akt also resulting in activation of the NF-kB, ${ }^{66}$ the central mediator of the inflammatory process and innate immunity. ${ }^{67}$ Except in regulating inflammatory responses, this signaling pathway may play an important role in autophagy. The combination of Akt inhibitor and chloroquine can significantly reduce the growth and regeneration of endometriosis stromal cells by inducing autophagy, ${ }^{68}$ certainly, the theoretical basis for this experiment is that PI3K/Akt signaling pathways play an important role in the disease, in fact, there is also surmise that the pathway is involved in progesterone antagonism. ${ }^{58}$ Moreover, the inhibition of the $\mathrm{PI3K} / \mathrm{Akt} / \mathrm{mTOR}$ signaling pathway may relieve endometriosis-associated sciatic nerve pain in a rat model of sciatic endometriosis.

IL-6 is a major pro-inflammatory cytokine that is synthesized as a $26 \mathrm{kDa}$ protein. In canonical IL- 6 signaling, IL- 6 first binds to the membrane-bound IL-6 receptor (mIL-6R) then gp130 dimerization results in the activation of tyrosine kinase Janus kinases 1,2 (JAK1,2). ${ }^{69}$ The transcription factor, STAT3 is localized in the cytoplasm until activated by phosphorylation. ${ }^{70,71}$ STAT3 is one of the major signal transducers of JAKs and plays a pivotal role in regulating many cellular functions such as cell differentiation and proliferation. ${ }^{72}$ Phosphorylated STAT3 is highly expressed in the endometrium of patients with endometriosis. Elevated pSTAT3 induces and stabilizes HIF1A within the eutopic endometrium of women with endometriosis. ${ }^{73}$ HIF1A has previously been shown to up-regulate many of the aberrant 
proteins and factors associated with endometriosis, including increased cell proliferation and viability, ${ }^{74}$ aberrant estrogen receptor-beta (ER $\beta$ ) expression, ${ }^{75,76}$ and increase vascular endothelial growth factor (VEGF). ${ }^{77} \mathrm{~A}$ study proved silencing HIF1A (siRNA) suppressed the expression of hypoxia-induced VEGF in endometrial cells. ${ }^{78}$

It can be seen from the above analysis that the molecular mechanism of LCP in the treatment of EM is mainly focused on regulating the inflammatory response, and the latest research results also show that inflammation plays an important role in this disease. ${ }^{79}$ In summary, through KEGG enrichment analysis, we sorted out the possible molecular mechanisms of LCP in the treatment of EM into four categories.1) Control inflammatory response. For example, this may reduce the reactivity of inflammatory factors by regulating TNF and HIF-1 signaling pathways and reduce adhesion, infiltration, growth, and shedding of ectopic focus. Also, ectopic tissue growth may be inhibited by regulating PD-L1 expression and PD-1 checkpoint pathway in cancer improving the immunity of the local tissue microenvironment. ${ }^{80}$ 2) Regulated hormone secretion. It may regulate estrogen signaling pathways to control EM progression by regulating HIF-1 signalingpathways.3) Inhibit cell proliferation and angiogenesis and growth. The progression of EM can be controlled by regulating VEGF signaling pathways or PI3K/Akt signaling pathways. 4) Regulation of neurotrophin signaling pathway relieve pelvic pain. In addition, by reading the literature, we found that the molecular biological characteristics of the eutopic endometrium play an important role in the development of EM. However, proteins post-translational modifications (PTMs) like include phosphorylation, acetylation, ubiquitination, and glycosylation, etc may be involved in the pathogenesis of EM, which leads to enhance the facility of adhesion, invasion, and angiogenesis in ectopic endometrium, making it more susceptible to get the disease. ${ }^{81}$ The molecular biological characteristics of the eutopic endometrium play an important role in determining the biological behavior of the ectopic endometrium, which is the classic "eutopic endometrium determinism" proposed by Lang Jing-he. ${ }^{82}$ It can categorize complex pathogenesis into monistic cognition. Based on this theory, the purpose of our research looks forward to finding key targets through network pharmacology and molecular docking will further promote the study of this disease.

\section{Conclusion}

We performed a network pharmacological analysis and molecular docking validation to discuss the therapeutic method of EM and explored the active components and mechanism of LCP. And the results fully show that the main active molecules in the treatment of EM and relief related symptoms in terms of anti-inflammation, promoting apoptosis, inhibiting angiogenesis, and relieving pain via the PI3K-Akt signaling pathway, HIF-1 signaling pathway, VEGF signaling pathway, estrogen signaling pathway, neurotrophin signaling, and the regulation of related protein such as RELAIEGFRIFOS, etc. However, there are still some limitations that should be noted. Firstly, the target research beyond molecular docking verification is not clear, in the next place, there is a lack of further experimental verification. It is hoped that the following clinical trials and basic research can further verify the molecular mechanism of LCP.

\section{Abbreviations}

EM: Endometriosis; LCP: Li Chong Pill; TCMSP: Traditional Chinese Medicine Systems Pharmacology Database and Analysis Platform

\section{Declarations}

\section{Ethics approval and informed consent}

Page 12/28 
Not applicable

\section{Consent for publication}

Not applicable.

\section{Data Availability}

The data used to support the result of this study can be obtained from the corresponding author.

\section{Funding Statement}

This study was supported by the National Natural Science Foundation of China (Grant. no. 82074484), (Grant.no.JDZX2015052), and Longjiang Scholars Program of Education Department of Heilongjiang Province (Grant.no.T201914)

\section{Competing interests}

The authors declare that they have no competing interests.

\section{Author contributions}

All authors contributed to data analysis, drafting or revising the article, gave final approval of the version to be published, Have agreed on the journal to which the article will be submitted, and agree to be accountable for all aspects of the work.

\section{Acknowledgments}

Not applicable.

\section{References}

1. Amreen S, Kumar P, Gupta P, Rao P. Evaluation of Oxidative Stress and Severity of Endometriosis. Journal of human reproductive sciences. Jan-Mar 2019;12(1):40-46. doi:10.4103/jhrs.JHRS_27_17

2. Chapron C, Marcellin L, Borghese B, Santulli P. Rethinking mechanisms, diagnosis and management of endometriosis. Nature reviews Endocrinology. Nov 2019;15(11):666-682. doi:10.1038/s41574-019-0245-z

3. Schwartz K, Llarena NC, Rehmer JM, Richards EG, Falcone T. The role of pharmacotherapy in the treatment of endometriosis across the lifespan. Expert opinion on pharmacotherapy. Jun 2020;21(8):893-903. doi:10.1080/14656566.2020.1738386

4. Sibiude J, Santulli P, Marcellin L, Borghese B, Dousset B, Chapron C. Association of history of surgery for endometriosis with severity of deeply infiltrating endometriosis. Obstetrics and gynecology. Oct 2014;124(4):709-717. doi:10.1097/aog.0000000000000464

5. Long Q, Liu X, Guo SW. Surgery accelerates the development of endometriosis in mice. American journal of obstetrics and gynecology. Sep 2016;215(3):320.e1-320.e15. doi:10.1016/j.ajog.2016.02.055

6. Weilin Z, Ziyan H, Lixing C, Yuanyuan M, Xuefang L. Therapeutic Thoughts and Methods of Endometriosis: Treatment Aiming at Its Root Causes. Journal of Traditional Chinese Medicine. 2016;57(09):737-739.

7. Ya-ru F, Wen-ting Z, Er-wen L, et al. Research progress on chemical constituents and pharmacological activities of Sparganium stoloniferum. Chinese Traditional and Herbal Drugs. 2017;48(22):4804-4818. 
8. Yongjian Z, Kai N, Dezhi T, et al. Research on Pharmacological Effects of Peach Kernel. Liaoning Journal of Traditional Chinese Medicine. 2015;42(04):888-890.

9. Lulu K, Haixia L, Yu S, Xuan L. Research about antitumor bio-activities of Rhizoma Sparganii and Rhizoma Curcumae. Jilin Journal of Chinese Medicine. 2017;37(07):722-724.

10. W. W, L. S, L. Q, L. H. Research progress on pharmacological effects of astragali radix-angelicae sinensis radix on invigorating qi and activating blood. Chinese Journal of Experimental Traditional Medical Formulae.1-10. doi:10.13422/j.cnki.syfjx.20210204

11. Dong H, Ren JX, Wang JJ, et al. Chinese Medicinal Leech: Ethnopharmacology, Phytochemistry, and Pharmacological Activities. Evidence-based complementary and alternative medicine : eCAM. 2016;2016:7895935. doi:10.1155/2016/7895935

12. Yanan Z, Shuai W, Yongrui B, Tianjiao L, Xiansheng M. Research of Different Medicinal Parts of Anemarrhena Asphodeloides on Anti-tumor, Anti-inflammatory and Anti-oxidation in vitro Based on Pharmacodynamics. Modernization of Traditional Chinese Medicine and Materia Medica-World Science and Technology. 2019;21(03):424-430.

13. Caixia W, Wene L, Minghui H. Analysis of ZHANG Xichun's Experience in Treating Women Abdominal Mass and the Modern Application. Clinical Journal of Traditional Chinese Medicine. 2019;31(11):2068-2070.

14. Yi-fei L, Yun S, Xian-zhi S, Wen-juan K, Xiao-mei W, Fei Y. Analysis of ZHANG Xi-chun's academic thought of gynecology and characteristics of medication. China Journal of Traditional Chinese Medicine and Pharmacy. 2020;35(07):3568-3571.

15. Luo TT, Lu Y, Yan SK, Xiao X, Rong XL, Guo J. Network Pharmacology in Research of Chinese Medicine Formula: Methodology, Application and Prospective. Chinese journal of integrative medicine. Jan 2020;26(1):72-80. doi:10.1007/s11655-019-3064-0

16. Pinzi L, Rastelli G. Molecular Docking: Shifting Paradigms in Drug Discovery. International journal of molecular sciences. Sep 4 2019;20(18)doi:10.3390/ijms20184331

17. Li Y-B, Huang W-H, Xiang Y. Three New Pteridines, Hirudinoidines A - C, from Hirudo nipponica Whitman. 2008;91(2):303-307. doi:https://doi.org/10.1002/hlca.200890035

18. Rongqing H, Chuanghuan L, Jiangnan P, et al. GC-MS Study on Active Components of Small Molecules in Leech. Chinese Traditional and Herbal Drugs. 2003;(09):23-24.

19. Wen-guang J, Jiang F, Yu-mei L, An L. Chemical Constituents of Hirudo. Chinese Journal of Experimental Traditional Medical Formulae. 2014;20(19):120-123.

20. B LY, Y. X, H HW, N Y. Chemical constituents from Hirudo nipponica Whitman. Strait Pharmaceutical Journal. 2009;21(05):75-77.

21. Xinyan Q. Study on anti-thrombin function of medicinal leech. Modern Journal of Integrated Traditional Chinese and Western Medicine. 2010;19(13):1582-1583.

22. Tang Y, Li M, Wang J, Pan Y, Wu FX. CytoNCA: a cytoscape plugin for centrality analysis and evaluation of protein interaction networks. Bio Systems. Jan 2015;127:67-72. doi:10.1016/j.biosystems.2014.11.005

23. Ashburner M, Ball CA, Blake JA, et al. Gene ontology: tool for the unification of biology. The Gene Ontology Consortium. Nature genetics. May 2000;25(1):25-9. doi:10.1038/75556

24. Ogata H, Goto S, Sato K, Fujibuchi W, Bono H, Kanehisa M. KEGG: Kyoto Encyclopedia of Genes and Genomes. Nucleic acids research. Jan 1 1999;27(1):29-34. doi:10.1093/nar/27.1.29

25. Peiris AN, Chaljub E, Medlock D. Endometriosis. Jama. Dec 25 2018;320(24):2608. doi:10.1001/jama.2018.17953 
26. Patel BG, Lenk EE, Lebovic DI, Shu Y, Yu J, Taylor RN. Pathogenesis of endometriosis: Interaction between Endocrine and inflammatory pathways. Best practice \& research Clinical obstetrics \& gynaecology. Jul 2018;50:50-60. doi:10.1016/j.bpobgyn.2018.01.006

27. Riccio L, Santulli P, Marcellin L, Abrão MS, Batteux F, Chapron C. Immunology of endometriosis. Best practice \& research Clinical obstetrics \& gynaecology. Jul 2018;50:39-49. doi:10.1016/j.bpobgyn.2018.01.010

28. Lin Y, Jia R, Liu Y, et al. Diosgenin inhibits superoxide generation in FMLP-activated mouse neutrophils via multiple pathways. Free radical research. Dec 2014;48(12):1485-93. doi:10.3109/10715762.2014.966705

29. Gao M, Chen L, Yu H, Sun Q, Kou J, Yu B. Diosgenin down-regulates NF-kB p65/p50 and p38MAPK pathways and attenuates acute lung injury induced by lipopolysaccharide in mice. International immunopharmacology. Feb 2013;15(2):240-5. doi:10.1016/j.intimp.2012.11.019

30. Jiaxi S, Li M, Junping K, Boyang Y. Diosgenin reduces leukocytes adhesion and migration linked with inhibition of intercellular adhesion molecule-1 expression and NF-KB p65 activation in endothelial cells. Chinese Journal of Natural Medicines. 2012;10(02):142-149.

31. Choi KW, Park HJ, Jung DH, et al. Inhibition of TNF-a-induced adhesion molecule expression by diosgenin in mouse vascular smooth muscle cells via downregulation of the MAPK, Akt and NF-KB signaling pathways. Vascular pharmacology. Nov-Dec 2010;53(5-6):273-80. doi:10.1016/j.vph.2010.09.007

32. Lee D, Kim SK, Lee JR, Jee BC. Management of endometriosis-related infertility: Considerations and treatment options. Clinical and experimental reproductive medicine. Mar 2020;47(1):1-11. doi:10.5653/cerm.2019.02971

33. Scutiero G, lannone P, Bernardi G, et al. Oxidative Stress and Endometriosis: A Systematic Review of the Literature. Oxidative medicine and cellular longevity. 2017;2017:7265238. doi:10.1155/2017/7265238

34. Gong G, Qin Y, Huang W. Anti-thrombosis effect of diosgenin extract from Dioscorea zingiberensis C.H. Wright in vitro and in vivo. Phytomedicine : international journal of phytotherapy and phytopharmacology. Apr 15 2011;18(6):458-63. doi:10.1016/j.phymed.2010.08.015

35. Gong G, Qin Y, Huang W, et al. Protective effects of diosgenin in the hyperlipidemic rat model and in human vascular endothelial cells against hydrogen peroxide-induced apoptosis. Chemico-biological interactions. Mar 30 2010;184(3):366-75. doi:10.1016/j.cbi.2010.02.005

36. Kiasalari Z, Rahmani T, Mahmoudi N, Baluchnejadmojarad T, Roghani M. Diosgenin ameliorates development of neuropathic pain in diabetic rats: Involvement of oxidative stress and inflammation. Biomedicine \& pharmacotherapy = Biomedecine \& pharmacotherapie. Feb 2017;86:654-661. doi:10.1016/j.biopha.2016.12.068

37. Madkour MM, Anbar HS, El-Gamal MI. Current status and future prospects of p38a/MAPK14 kinase and its inhibitors. European journal of medicinal chemistry. Jan 22 2021;213:113216.

doi:10.1016/j.ejmech.2021.113216

38. Schenone S, Manetti F, Botta M. SRC inhibitors and angiogenesis. Current pharmaceutical design. 2007;13(21):2118-28. doi:10.2174/138161207781039580

39. Arafeh R, Samuels Y. PIK3CA in cancer: The past 30 years. Seminars in cancer biology. Dec 2019;59:36-49. doi:10.1016/j.semcancer.2019.02.002

40. Bulun SE, Wan Y, Matei D. Epithelial Mutations in Endometriosis: Link to Ovarian Cancer. Endocrinology. Mar 1 2019;160(3):626-638. doi:10.1210/en.2018-00794

41. Cao Y, Zhuang MF, Yang Y, et al. Preliminary study of quercetin affecting the hypothalamic-pituitary-gonadal axis on rat endometriosis model. Evidence-based complementary and alternative medicine : eCAM.

2014;2014:781684. doi:10.1155/2014/781684

Page 15/28 
42. Park S, Lim W, Bazer FW, Whang KY, Song G. Quercetin inhibits proliferation of endometriosis regulating cyclin D1 and its target microRNAs in vitro and in vivo. The Journal of nutritional biochemistry. Jan 2019;63:87-100. doi:10.1016/j.jnutbio.2018.09.024

43. Devi KP, Malar DS, Nabavi SF, et al. Kaempferol and inflammation: From chemistry to medicine. Pharmacological research. Sep 2015;99:1-10. doi:10.1016/j.phrs.2015.05.002

44. Gong G, Guan YY, Zhang ZL, et al. Isorhamnetin: A review of pharmacological effects. Biomedicine \& pharmacotherapy = Biomedecine \& pharmacotherapie. Aug 2020;128:110301. doi:10.1016/j.biopha.2020.110301

45. Ren X, Han L, Li Y, et al. Isorhamnetin attenuates TNF-a-induced inflammation, proliferation, and migration in human bronchial epithelial cells via MAPK and NF-KB pathways. Anatomical record (Hoboken, NJ : 2007). Aug 31 2020;doi:10.1002/ar.24506

46. Zhou B, Rao L, Peng Y, et al. A functional promoter polymorphism in NFKB1 increases susceptibility to endometriosis. DNA and cell biology. May 2010;29(5):235-9. doi:10.1089/dna.2009.0992

47. Huang S, Robinson JB, Deguzman A, Bucana CD, Fidler IJ. Blockade of nuclear factor-kappaB signaling inhibits angiogenesis and tumorigenicity of human ovarian cancer cells by suppressing expression of vascular endothelial growth factor and interleukin 8. Cancer research. Oct 1 2000;60(19):5334-9.

48. Haining RE, Cameron IT, van Papendorp C, et al. Epidermal growth factor in human endometrium: proliferative effects in culture and immunocytochemical localization in normal and endometriotic tissues. Human reproduction (Oxford, England). Oct 1991;6(9):1200-5. doi:10.1093/oxfordjournals.humrep.a137512

49. Rakhila $\mathrm{H}, \mathrm{Al}$-Akoum M, Bergeron ME, et al. Promotion of angiogenesis and proliferation cytokines patterns in peritoneal fluid from women with endometriosis. Journal of reproductive immunology. Aug 2016;116:1-6. doi:10.1016/j.jri.2016.01.005

50. Zhan H, Peng B, Ma J, et al. Epidermal growth factor promotes stromal cells migration and invasion via upregulation of hyaluronate synthase 2 and hyaluronan in endometriosis. Fertility and sterility. Oct 2020;114(4):888-898. doi:10.1016/j.fertnstert.2020.05.005

51. Yang H, Liu J, Fan Y, et al. Associations between various possible promoter polymorphisms of MMPs genes and endometriosis risk: a meta-analysis. European journal of obstetrics, gynecology, and reproductive biology. Oct 2016;205:174-88. doi:10.1016/j.ejogrb.2016.08.015

52. Chatterjee K, Jana S, DasMahapatra P, Swarnakar S. EGFR-mediated matrix metalloproteinase-7 up-regulation promotes epithelial-mesenchymal transition via ERK1-AP1 axis during ovarian endometriosis progression. FASEB journal : official publication of the Federation of American Societies for Experimental Biology. Aug 2018;32(8):4560-4572. doi:10.1096/fj.201701382RR

53. Dong-ping T, Xiao-jun M, Chang-ming M, Xi-jun Q, Jie H. Progress and Bioinformatics Analysis of FOS Protein. Hubei Agricultural Sciences. 2015;54(07):1537-1542.

54. Bulun SE. Endometriosis. The New England journal of medicine. Jan 15 2009;360(3):268-79. doi:10.1056/NEJMra0804690

55. Reis FM, Maia AL, Ribeiro MF, Spritzer PM. Progestin modulation of c-fos and prolactin gene expression in the human endometrium. Fertility and sterility. Jun 1999;71(6):1125-32. doi:10.1016/s0015-0282(99)00138-7

56. Pan H, Zhang P, Li JR, et al. c-Fos-Regulated Matrix Metalloproteinase-9 Expression is Involved in 17 $\beta$-EstradiolPromoted Invasion of Human Endometrial Stromal Cell. Current molecular medicine. 2016;16(3):266-75. doi:10.2174/1566524016666160225153454 
57. Yilmaz BD, Bulun SE. Endometriosis and nuclear receptors. Human reproduction update. Jul 1 2019;25(4):473485. doi:10.1093/humupd/dmz005

58. Lee, II, Kim JJ. Influence of AKT on progesterone action in endometrial diseases. Biology of reproduction. Sep 2014;91(3):63. doi:10.1095/biolreprod.114.119255

59. Bui M, Hao X, Shin Y, et al. Synthesis and SAR study of potent and selective PI3Kס inhibitors. Bioorganic \& medicinal chemistry letters. Mar 1 2015;25(5):1104-9. doi:10.1016/j.bmcl.2015.01.001

60. Allen RA, Brookings DC, Powell MJ, et al. Seletalisib: Characterization of a Novel, Potent, and Selective Inhibitor of PI3Kס. The Journal of pharmacology and experimental therapeutics. Jun 2017;361(3):429-440. doi:10.1124/jpet.116.237347

61. Cheung TT, Mclnnes IB. Future therapeutic targets in rheumatoid arthritis? Seminars in immunopathology. Jun 2017;39(4):487-500. doi:10.1007/s00281-017-0623-3

62. Courtnay R, Ngo DC, Malik N, Ververis K, Tortorella SM, Karagiannis TC. Cancer metabolism and the Warburg effect: the role of HIF-1 and PI3K. Molecular biology reports. Apr 2015;42(4):841-51. doi:10.1007/s11033-0153858-x

63. Carnero A, Blanco-Aparicio C, Renner O, Link W, Leal JF. The PTEN/PI3K/AKT signalling pathway in cancer, therapeutic implications. Current cancer drug targets. May 2008;8(3):187-98. doi:10.2174/156800908784293659

64. Cianciulli A, Calvello R, Porro C, Trotta T, Salvatore R, Panaro MA. PI3k/Akt signalling pathway plays a crucial role in the anti-inflammatory effects of curcumin in LPS-activated microglia. International immunopharmacology. Jul 2016;36:282-290. doi:10.1016/j.intimp.2016.05.007

65. Xue JF, Shi ZM, Zou J, Li XL. Inhibition of PI3K/AKT/mTOR signaling pathway promotes autophagy of articular chondrocytes and attenuates inflammatory response in rats with osteoarthritis. Biomedicine \& pharmacotherapy = Biomedecine \& pharmacotherapie. May 2017;89:1252-1261. doi:10.1016/j.biopha.2017.01.130

66. Bai D, Ueno L, Vogt PK. Akt-mediated regulation of NFkappaB and the essentialness of NFkappaB for the oncogenicity of PI3K and Akt. International journal of cancer. Dec 15 2009;125(12):2863-70. doi:10.1002/ijc. 24748

67. Torrealba N, Vera R, Fraile B, Martínez-Onsurbe P, Paniagua R, Royuela M. TGF- $\beta / P I 3 K / A K T / m T O R / N F-k B$ pathway. Clinicopathological features in prostate cancer. The aging male : the official journal of the International Society for the Study of the Aging Male. Apr 11 2019:1-11. doi:10.1080/13685538.2019.1597840

68. Matsuzaki S, Pouly JL, Canis M. In vitro and in vivo effects of MK2206 and chloroquine combination therapy on endometriosis: autophagy may be required for regrowth of endometriosis. British journal of pharmacology. May 2018;175(10):1637-1653. doi:10.1111/bph.14170

69. Uciechowski P, Dempke WCM. Interleukin-6: A Masterplayer in the Cytokine Network. Oncology. 2020;98(3):131137. doi:10.1159/000505099

70. Heinrich PC, Behrmann I, Haan S, Hermanns HM, Müller-Newen G, Schaper F. Principles of interleukin (IL)-6-type cytokine signalling and its regulation. The Biochemical journal. Aug 15 2003;374(Pt 1):1-20. doi:10.1042/bj20030407

71. Zhong Z, Wen Z, Darnell JE, Jr. Stat3: a STAT family member activated by tyrosine phosphorylation in response to epidermal growth factor and interleukin-6. Science (New York, NY). Apr 1 1994;264(5155):95-8. doi:10.1126/science.8140422

72. Yeung YT, Aziz F, Guerrero-Castilla A, Arguelles S. Signaling Pathways in Inflammation and Anti-inflammatory Therapies. Current pharmaceutical design. 2018;24(14):1449-1484. doi:10.2174/1381612824666180327165604 
73. Kim BG, Yoo JY, Kim TH, et al. Aberrant activation of signal transducer and activator of transcription-3 (STAT3) signaling in endometriosis. Human reproduction (Oxford, England). May 2015;30(5):1069-78. doi:10.1093/humrep/dev050

74. Semenza GL. Hypoxia, clonal selection, and the role of HIF-1 in tumor progression. Critical reviews in biochemistry and molecular biology. 2000;35(2):71-103. doi:10.1080/10409230091169186

75. Juhasz-Böss I, Fischer C, Lattrich $C$, et al. Endometrial expression of estrogen receptor $\beta$ and its splice variants in patients with and without endometriosis. Archives of gynecology and obstetrics. Oct 2011;284(4):885-91. doi:10.1007/s00404-010-1768-7

76. Wu MH, Lu CW, Chang FM, Tsai SJ. Estrogen receptor expression affected by hypoxia inducible factor-1a in stromal cells from patients with endometriosis. Taiwanese journal of obstetrics \& gynecology. Mar 2012;51(1):50-4. doi:10.1016/j.tjog.2012.01.010

77. Tsuzuki T, Okada $\mathrm{H}$, Cho H, et al. Hypoxic stress simultaneously stimulates vascular endothelial growth factor via hypoxia-inducible factor-1 $a$ and inhibits stromal cell-derived factor-1 in human endometrial stromal cells. Human reproduction (Oxford, England). Feb 2012;27(2):523-30. doi:10.1093/humrep/der405

78. Maybin JA, Hirani N, Brown P, Jabbour HN, Critchley HO. The regulation of vascular endothelial growth factor by hypoxia and prostaglandin F『a during human endometrial repair. The Journal of clinical endocrinology and metabolism. Aug 2011;96(8):2475-83. doi:10.1210/jc.2010-2971

79. Samimi M, Pourhanifeh MH, Mehdizadehkashi A, Eftekhar T, Asemi Z. The role of inflammation, oxidative stress, angiogenesis, and apoptosis in the pathophysiology of endometriosis: Basic science and new insights based on gene expression. Journal of cellular physiology. Nov 2019;234(11):19384-19392. doi:10.1002/jcp.28666

80. Walankiewicz M, Grywalska E, Polak G, et al. The Increase of Circulating PD-1- and PD-L1-Expressing Lymphocytes in Endometriosis: Correlation with Clinical and Laboratory Parameters. Mediators of inflammation. 2018;2018:7041342. doi:10.1155/2018/7041342

81. Xu HM, Deng HT, Liu CD, Chen YL, Zhang ZY. Phosphoproteomics Analysis of Endometrium in Women with or without Endometriosis. Chinese medical journal. Oct 5 2015;128(19):2617-24. doi:10.4103/0366-6999.166022

82. Jing-he L. Past history,current situation and progress in the knowledge of endometriosi. Chinese Journal of Practical Gynecology and Obstetrics. 2020;36(03):193-196.

83. Ru J, Li P, Wang J, et al. TCMSP: a database of systems pharmacology for drug discovery from herbal medicines. Journal of cheminformatics. 2014;6:13. doi:10.1186/1758-2946-6-13

84. Xu L, Zhang J, Wang Y, Zhang Z, Wang F, Tang X. Uncovering the mechanism of Ge-Gen-Qin-Lian decoction for treating ulcerative colitis based on network pharmacology and molecular docking verification. Bioscience reports. Jan 7 2021;doi:10.1042/bsr20203565

85. Fishilevich S, Zimmerman S, Kohn A, et al. Genic insights from integrated human proteomics in GeneCards. Database : the journal of biological databases and curation. 2016;2016doi:10.1093/database/baw030

86. Amberger JS, Hamosh A. Searching Online Mendelian Inheritance in Man (OMIM): A Knowledgebase of Human Genes and Genetic Phenotypes. Current protocols in bioinformatics. Jun 27 2017;58:1.2.1-1.2.12. doi:10.1002/cpbi.27

87. Qin C, Zhang C, Zhu F, et al. Therapeutic target database update 2014: a resource for targeted therapeutics. Nucleic acids research. Jan 2014;42(Database issue):D1118-23. doi:10.1093/nar/gkt1129

88. Wishart DS, Wu A. Using DrugBank for In Silico Drug Exploration and Discovery. Current protocols in bioinformatics. Jun 20 2016;54:14.4.1-14.4.31. doi:10.1002/cpbi.1 
89. Barbarino JM, Whirl-Carrillo M, Altman RB, Klein TE. PharmGKB: A worldwide resource for pharmacogenomic information. Wiley interdisciplinary reviews Systems biology and medicine. Jul 2018;10(4):e1417. doi:10.1002/wsbm.1417

90. Rabbani G, Baig MH, Ahmad K, Choi I. Protein-protein Interactions and their Role in Various Diseases and their Prediction Techniques. Current protein \& peptide science. 2018;19(10):948-957. doi:10.2174/1389203718666170828122927

91. Szklarczyk D, Gable AL, Lyon D, et al. STRING v11: protein-protein association networks with increased coverage, supporting functional discovery in genome-wide experimental datasets. Nucleic acids research. Jan 8 2019;47(D1):D607-d613. doi:10.1093/nar/gky1131

92. Burley SK, Berman HM, Kleywegt GJ, Markley JL, Nakamura H, Velankar S. Protein Data Bank (PDB): The Single Global Macromolecular Structure Archive. Methods in molecular biology (Clifton, NJ). 2017;1607:627-641. doi:10.1007/978-1-4939-7000-1_26

\section{Figures}




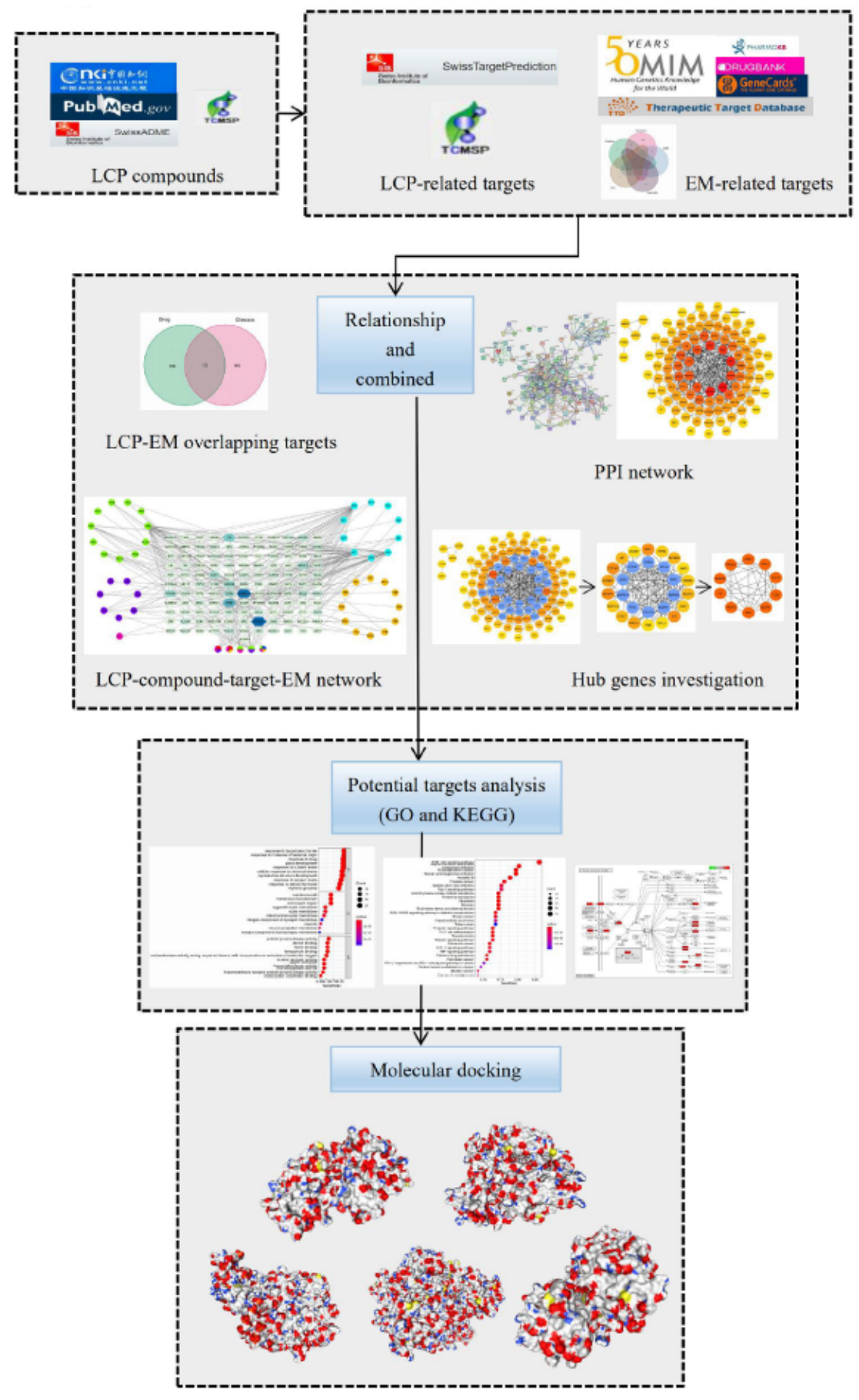

Figure 1

Technology route for exploring molecular mechanisms of LCP against EM based on network pharmacology and molecular docking 


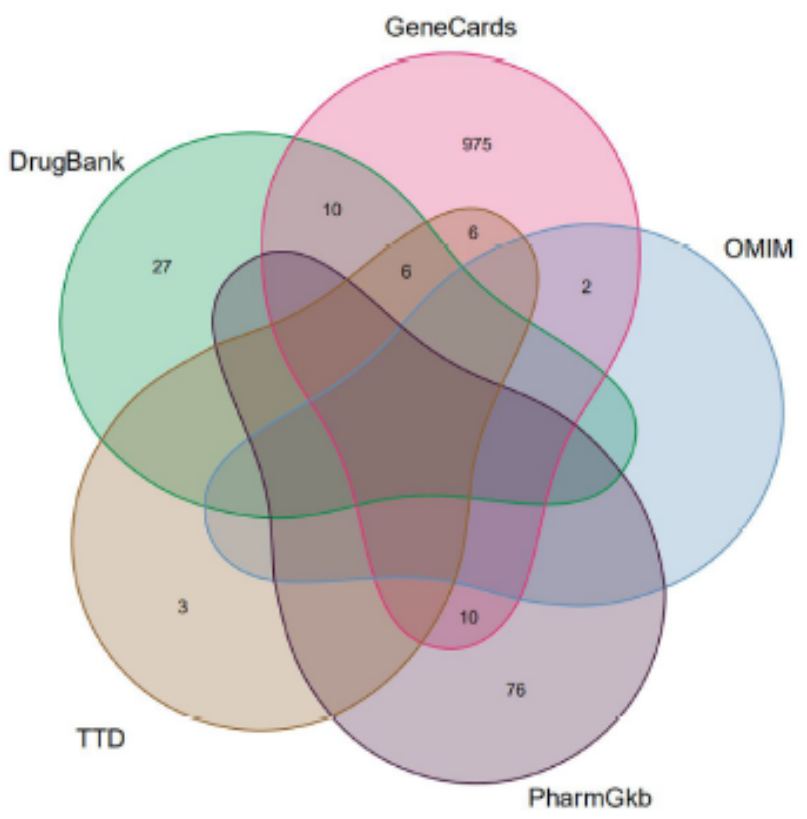

\section{Figure 2}

Venn diagram. Showing the EM-related targets of the overlapped and the specific genes among the five databases (OMIM, DrugBank, TTD, GeneCards, and PharmGKB).

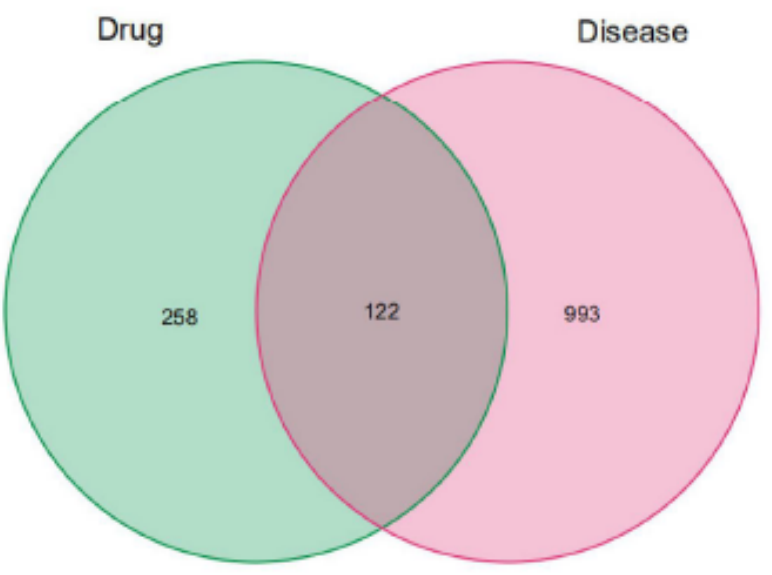

\section{Figure 3}

Venn diagram, 122 overlapping targets between LCP-related targets and EM-related targets. 


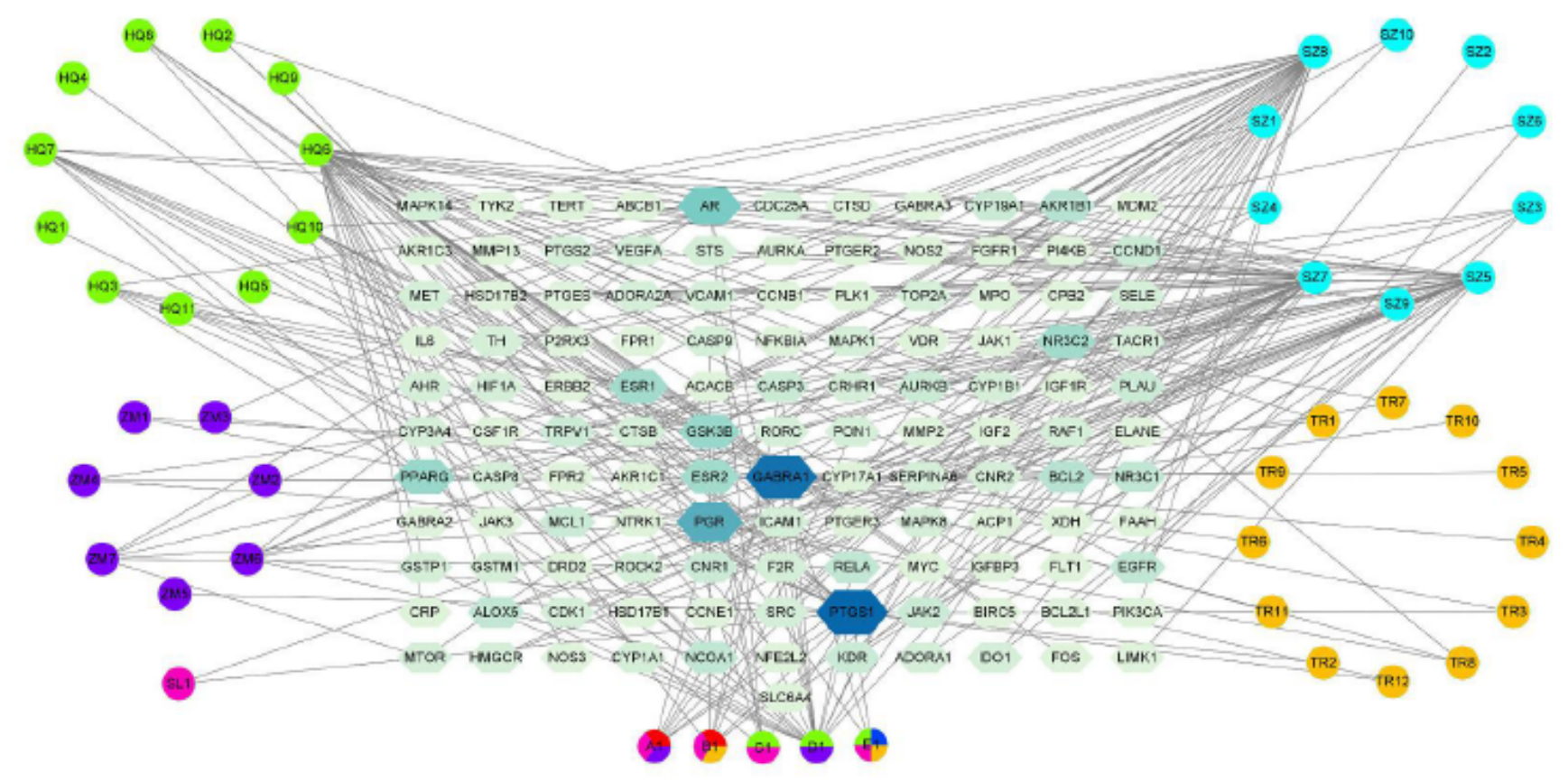

Figure 4

LCP-compound-target-EM Network (The blue pentagons represent 122 overlapping targets, different color means different herbs. Green represents Huangqi, blue represents Shuizhi, orange represents Taoren, red represents Danggui, pink represents Sanleng, indigo represents Zhimu). The edge between two nodes represented the interaction, the size and the gradation in color of each target node indicates the degree of connections. 


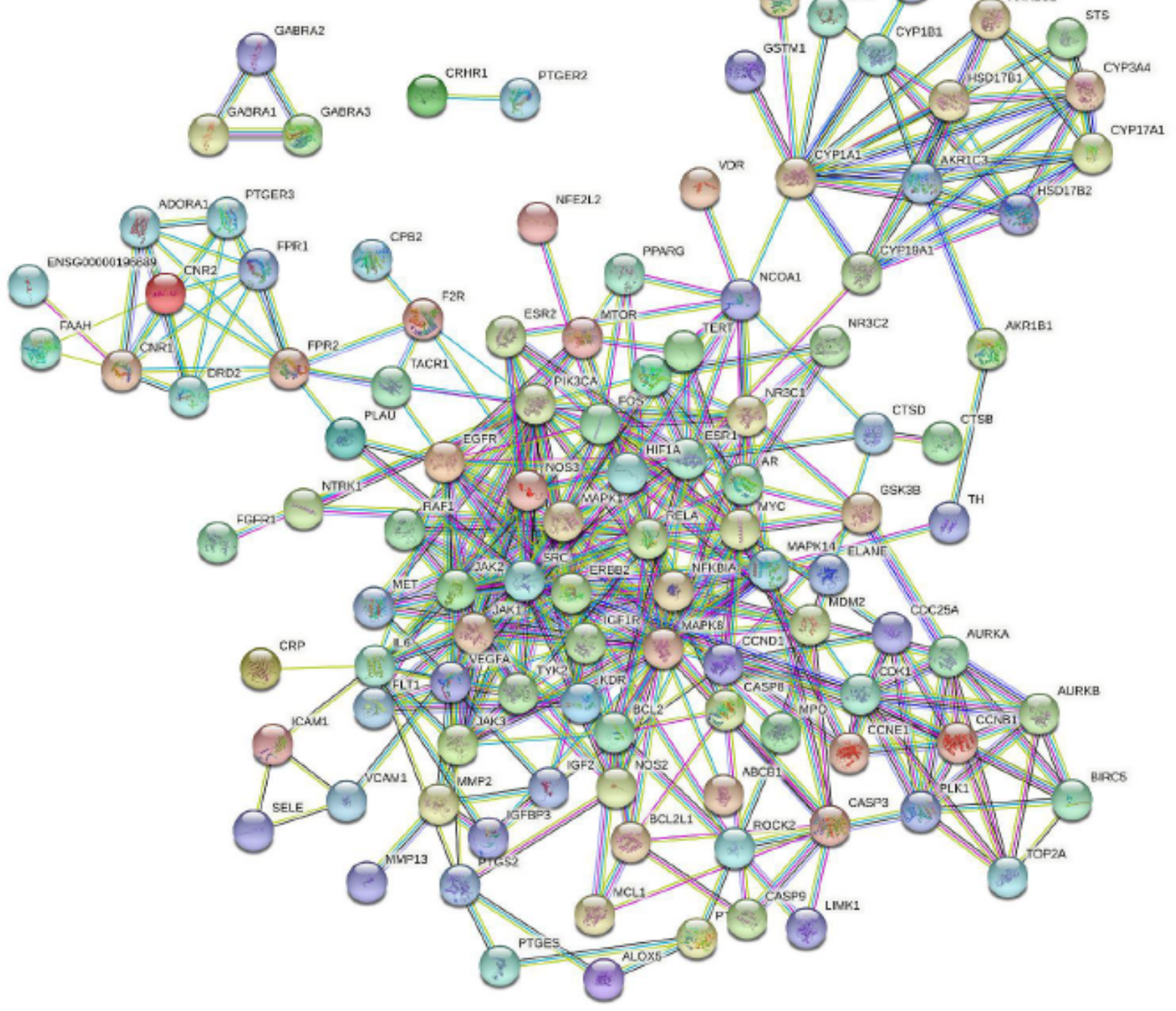

Figure 5

PPI network diagram from STRING protein database. 


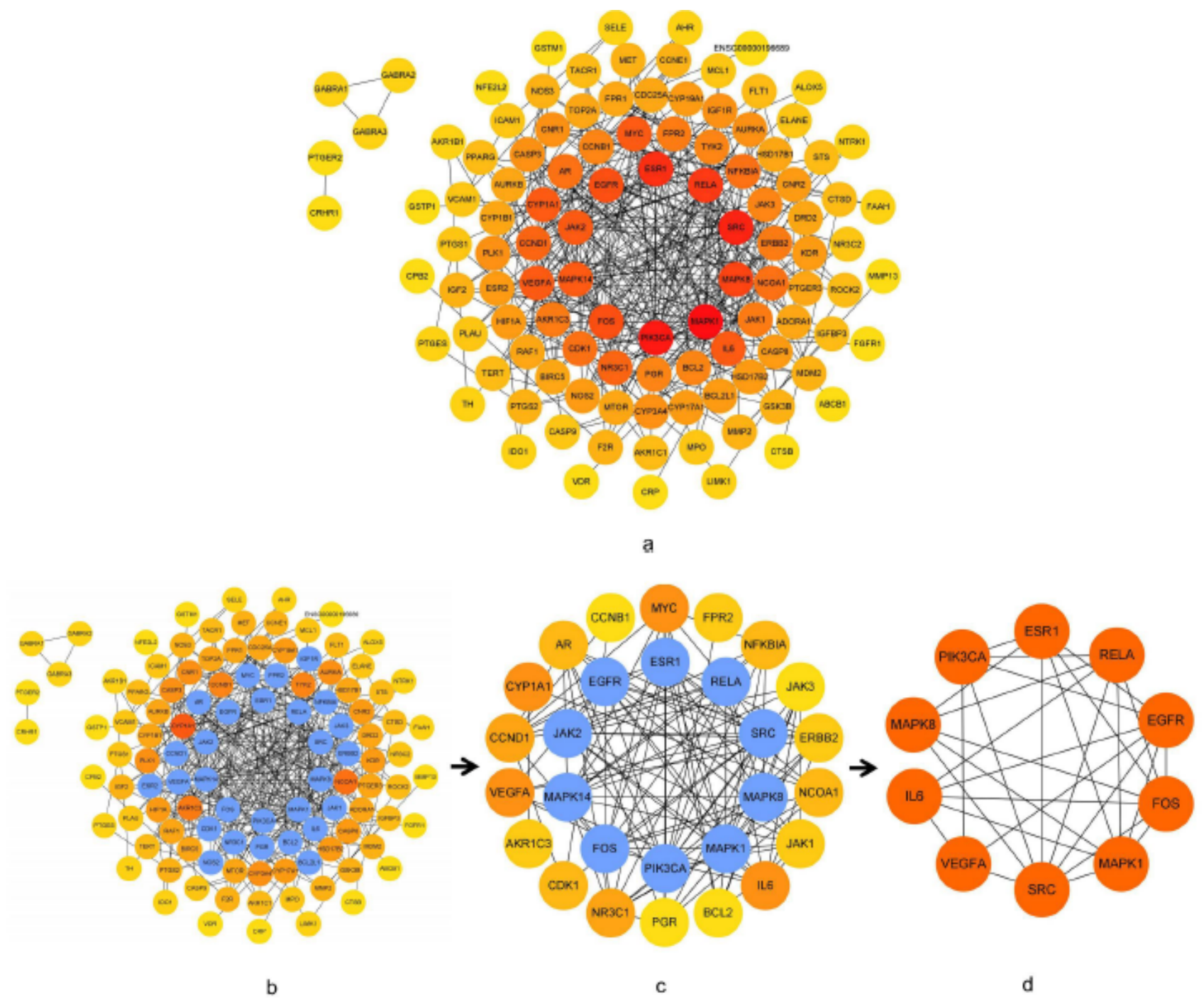

\section{Figure 6}

(a) PPI network related to potential targets. (b/c) Module analysis of the network for the identification of potential targets, the orange color indicates primary proteins, and the blue color indicates the next group proteins screened by betweeness, closeness, degree, eigenvector, LAC and network. (d) 10 highly connected nodes significant endometriosis-related targets. 


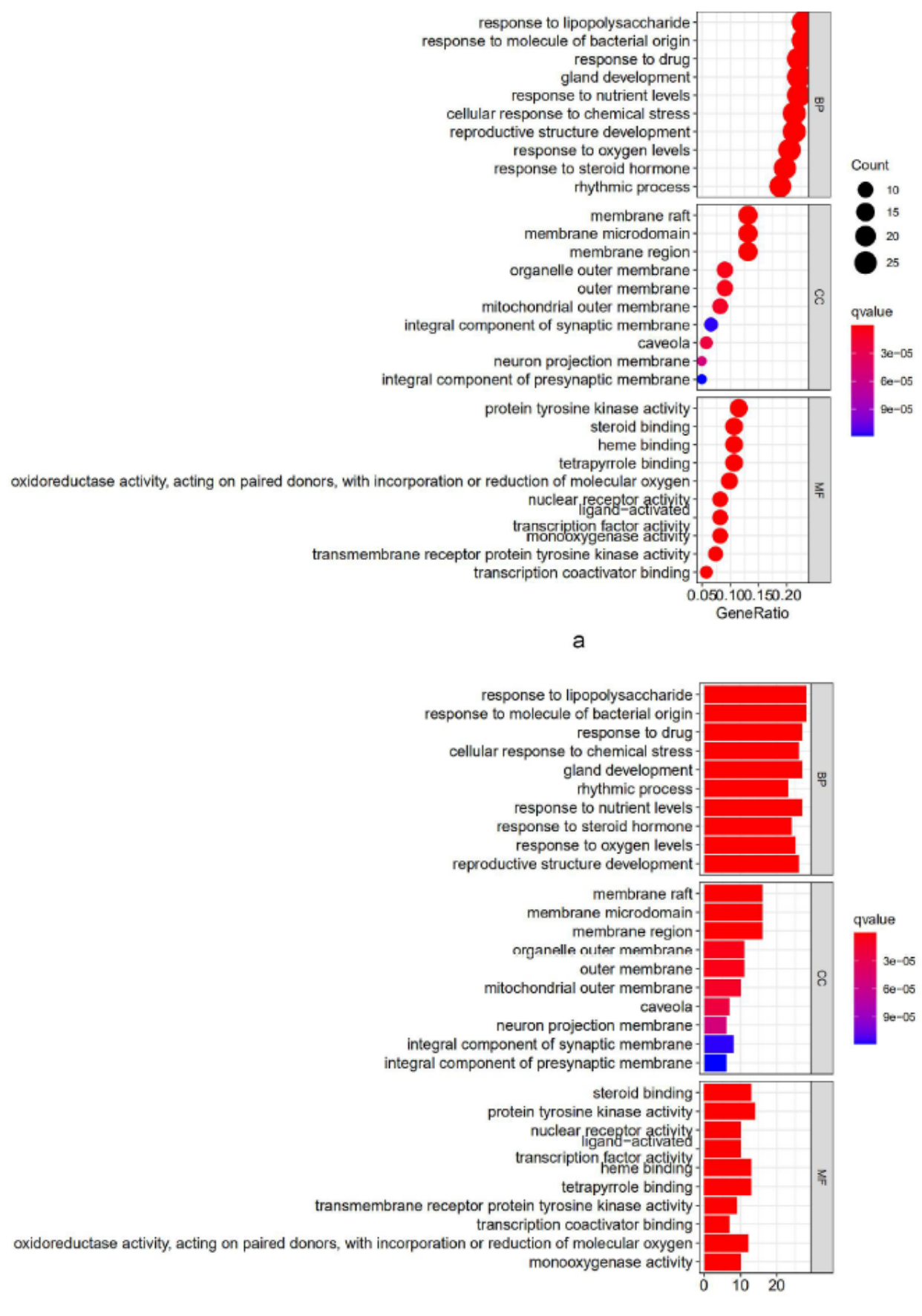

b

\section{Figure 7}

The results of Go (a/b) enrichment analysis.Picture (a) is bubble chart while the (b) is histogram. The abscissa and the circle size represent the enriched gene ratio, the gradation of color means enriched adjust P-value. 


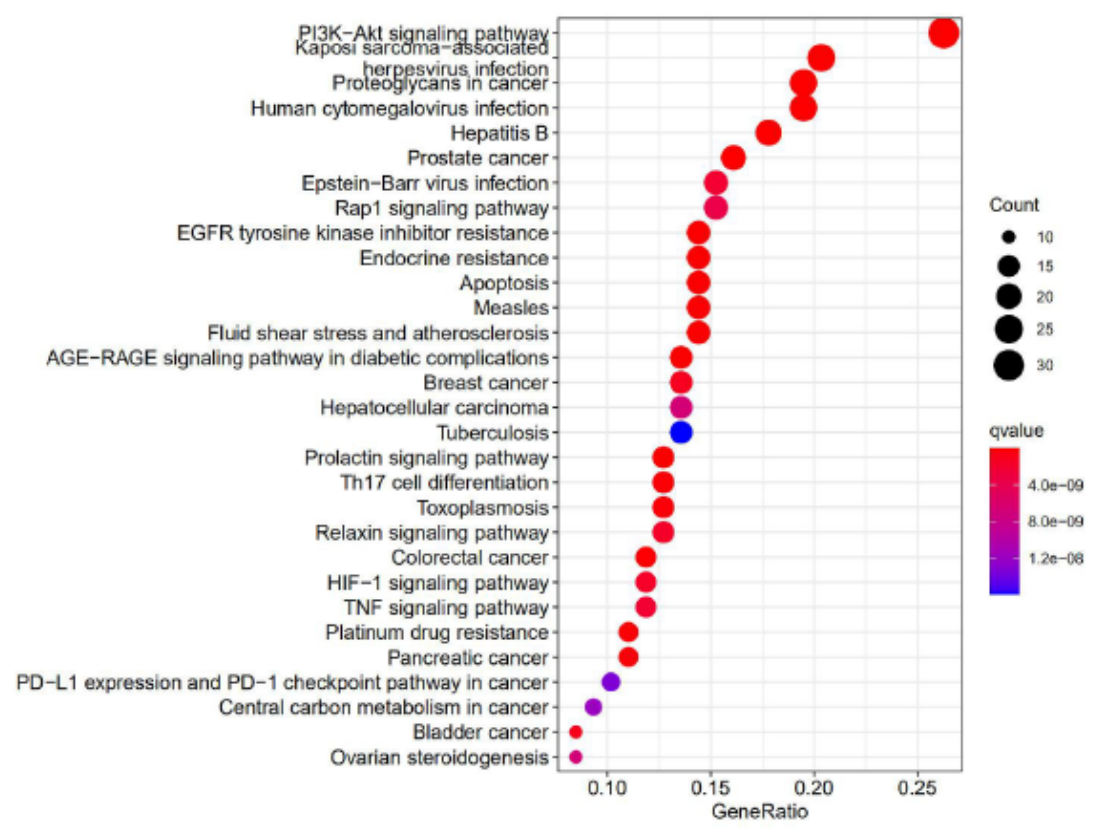

a

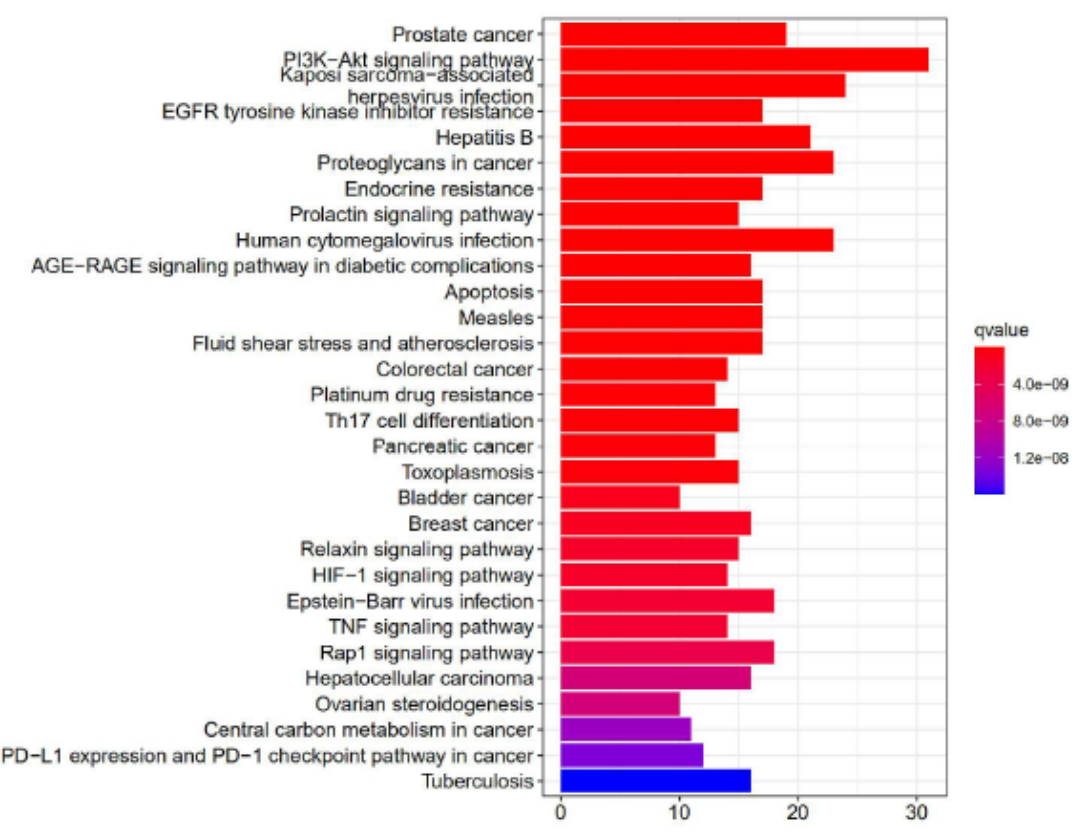

b

\section{Figure 8}

The results of KEGG (a/b) enrichment analysis.Picture (a) is bubble chart while the (b) is histogram. The abscissa and the circle size represent the enriched gene ratio, the gradation of color means enriched adjust P-value. 

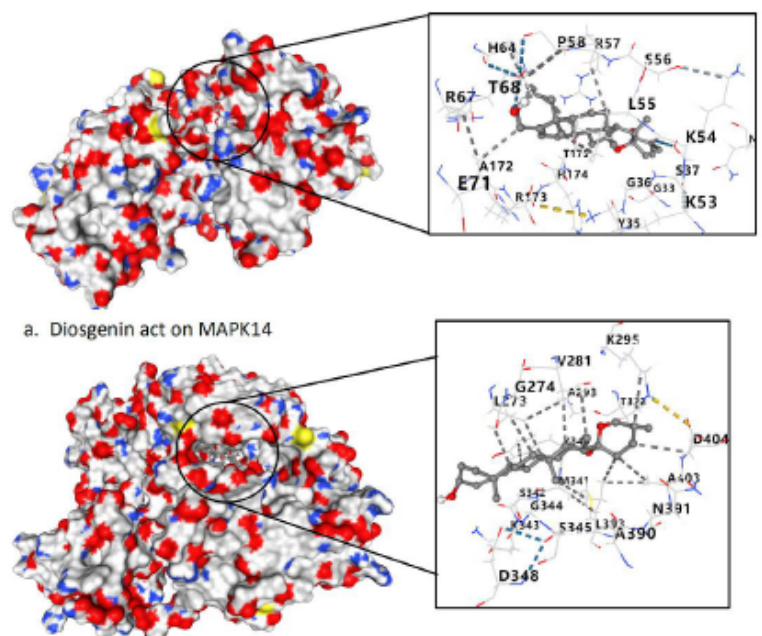

b. Diosgenin act on SRC

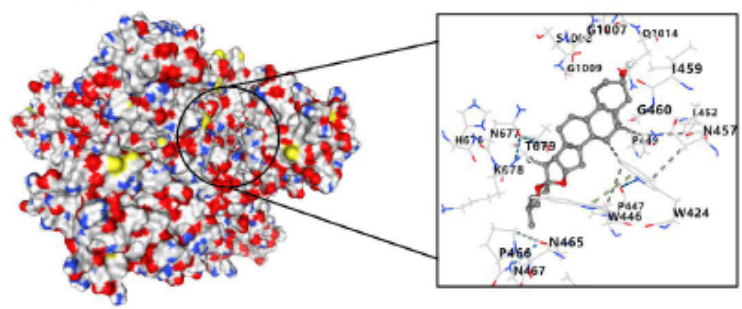

c. Diosgenin act on PIK3CA
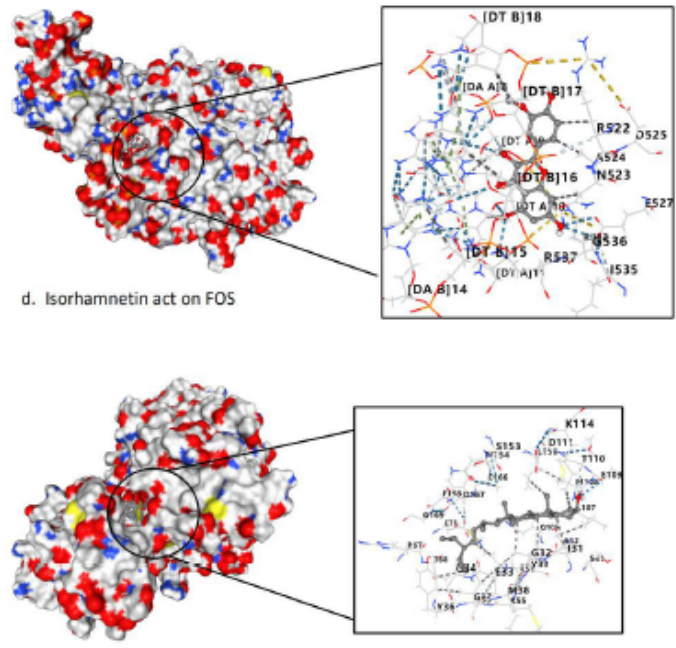

e. Isorhamnetin act on FOS

\section{Figure 9}

Detailed target-compound interactions with the five highest molecular docking affinity. 


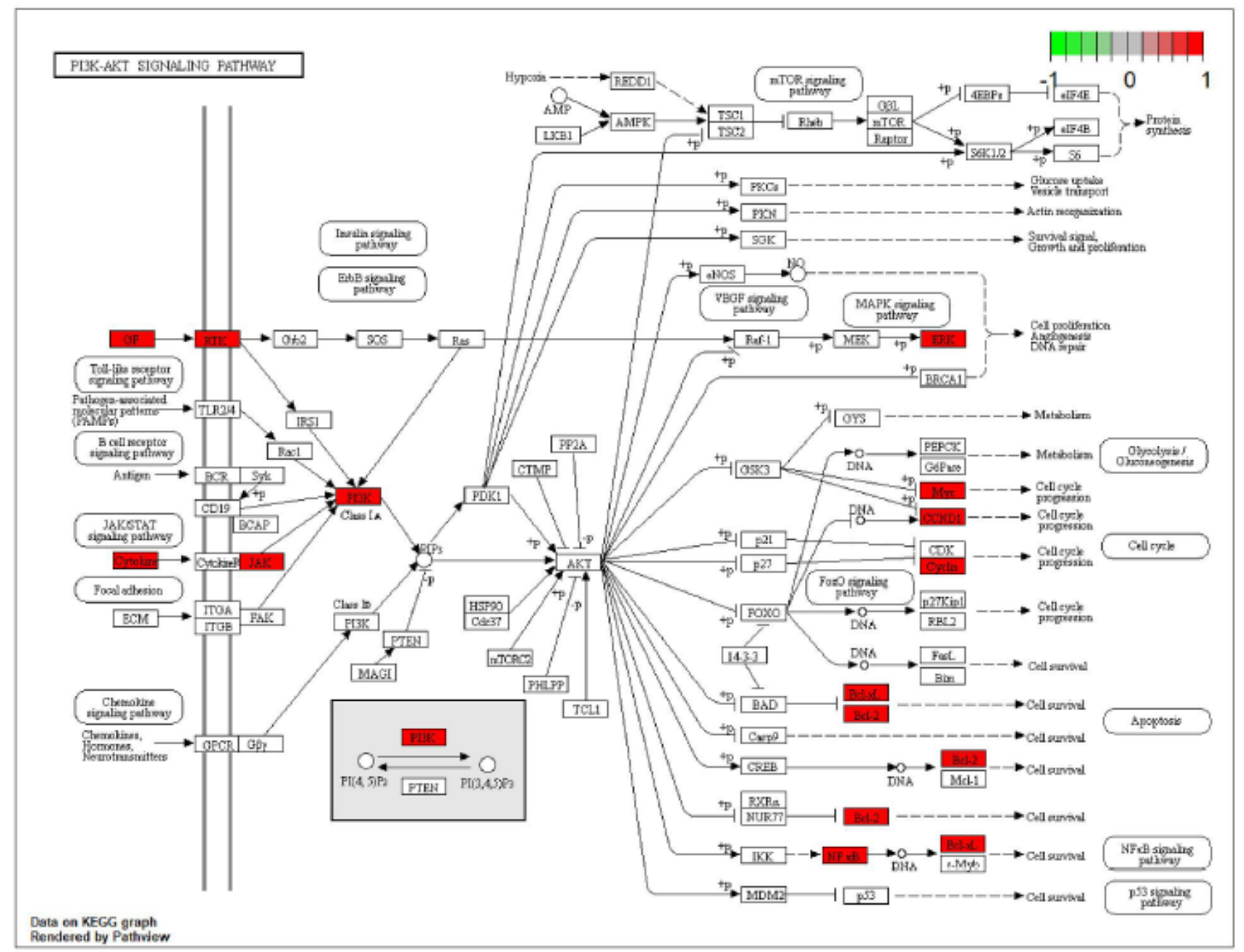

Figure 10

KEGG analysis pathway map (PI3K/Akt signaling pathway) of potential targets.

\section{Supplementary Files}

This is a list of supplementary files associated with this preprint. Click to download.

- Additionalfile1.xls

- Additionalfile2.xls

- Additionalfile3.xls

- Additionalfile4.xls

- Additionalfile5.xls

- Additionalfile6.xls 